\title{
Slow and Fast Light in Optical Fibers: Review and Perspectives
}

\author{
Luc Thévenaz \\ Ecole Polytechnique Fédérale de Lausanne, Institute of Electrical Engineering, STI-GR-SCI Station 11, 1015 Lausanne, Switzerland \\ luc.thevenaz@epfl.ch
}

\begin{abstract}
Fiber slow light systems are at a turning point moving from a laboratory research to real applications. The possibility to shape the spectral resonance in Brillouin slow light leads to optimized configurations and innovative solutions.

(C) 2009 Optical Society of America

OCIS Codes: (060.4370) Nonlinear optics, fibers ; (190.5890) Scattering, stimulated .
\end{abstract}

\section{Introduction}

The possibility to realize slow \& fast light elements in optical fibers has been considered like a an essential step moving slow \& fast light experiments from a scientific curiosity to the real world, since such elements can be seamlessly integrated in all fiber-based systems [1]. Since the pioneering experiments in 2004-2005 using the sharp spectral resonance induced by stimulated Brillouin scattering $[2,3]$ steady progresses have been realized towards longer delays [4], higher bit rates [5], lower distortion [6] and eventually the compatibility with telecommunication lines [7]. In this presentation the state-of-the art of slow \& fast light in optical fibers will be reviewed and an overview on future perspectives and trends in research will be discussed.

\section{Approaches for slow light in optical fibers}

In most implementation of slow \& fast light in optical fibers a spectral resonance is induced in the fiber medium through a parametric process that requires a strict phase matching condition. This secures a narrowband interaction. This resonance will in turn induce the sharp refractive index change that leads to the important and controllable group velocity change.

The most efficient parametric process in optical fibers - that turns out to be moreover very narrowband by essence - is stimulated Brillouin scattering (SBS), in which an energy transfer between 2 contrapropagating waves in a single mode fiber can be realized through the intercession of an acoustic wave. Efficient narrowband amplification can be realized, with milliwatt pump power and over a bandwidth of some $30 \mathrm{MHz}$. The first demonstrations of slow and fast light in optical fibers were logically realized using SBS [2,3], and this approach has steadily shown the best potentialities for the optimization of slow light systems, as described in the next section. The SBS implementation could also succeed in realizing important velocity changes in meter-long segments of optical fiber, from a 10-fold reduction of the group velocity [8] to acceleration up to a superluminal velocity and even a negative velocity [9].

Another approach based on the parametric amplification generated by the Kerr optical nonlinearity [10] was also proposed shortly after, that presents the advantage to show a bandwidth more compatible with the very high data rate exceeding $10 \mathrm{Gbit} / \mathrm{s}$, however at the expense of a pump power in the Watt range. In this approach the bandwidth can be modified to a large extent by judiciously placing pump and signal in the fiber dispersion curve.

Finally a fiber slow light element was also demonstrated based on stimulated scattering Raman, however the broadband nature of the Raman interaction restricts the operating range to the femtosecond scale [11].

Let's mention other approaches based on other concepts but parametric process, such as coherent population oscillation in erbium doped fibers [12], very efficient but restricted to kilohertz bandwidth, and gap solitons in fiber Bragg gratings [13].

\section{Engineered slow light in fiber delay lines}

A unique feature of SBS is the possibility to modify and freely shape the gain spectral distribution by modulating the pump spectrum [1]. This capability was soon identified like the solution to extend the bandwidth arbitrarily beyond the $30 \mathrm{MHz}$ given by the natural Brillouin linewidth [14]. The compatibility of Brillouin slow light systems with large bandwidth was then demonstrated up to $12 \mathrm{GHz}$ using a single pump [5] and even extended up to $25 \mathrm{GHz}$ using two pumps [15]. It should be mentioned that the required pump power increases linearly with the bandwidth to maintain the same normalized delay, leading to pump levels close to the Watt range for Gbit/s delay line.

But the possibility to shape the gain spectrum was not only used to extend the bandwidth, it turned out to be also a good solution to make the slowing effect more efficient (more delay for an equivalent pump power) and with less 


\section{JTuE1.pdf}

distortion [16]. It could be demonstrated that a rectangular gain spectrum is the optimized spectral distribution to minimize distortion for a given delay $[6,17]$.

Very interesting and imaginative solutions have also been proposed by combining and superposing SBS gain and loss spectra, showing different spectral widths and/or central frequencies [1]. Following this approach delay lines with zero amplitude change could be demonstrated [18] and a substantial enhancement of the delaying efficiency could be realized [19].

\section{Perspectives}

Fiber slow light systems have now proved to be mature enough to be implemented in real systems; however they remain subject to fundamental limitations. Simple theoretical considerations show that the delaying effect naturally gives rise to a pulse broadening due to the combined effect of the low pass filtering in the spectral resonance and the residual third order dispersion induced by the sharp index change [17]. Even after optimization [6] the distortion remains acceptable only for few bits delay, far from the expectation for optical buffering in digital delay lines. Recent works have proposed some miraculous implementations to cancel broadening, but are mostly based on incomplete and biased views about the essence of the broadening effects. It remains that fiber slow light delay lines find an excellent field of application in analog signal processing, such as microwave photonics. For this class of application a one-period delay is sufficient for a full control and the inherent linearity of SBS slow light elements is a key asset.

Other research efforts are now carried out to find other fields of application for slow light that are not strictly related to a simple delaying effect. Currently few applications use the property that the light signal envelope intrinsically propagates at a lower velocity. Some studies show that slow light may be beneficial to enhance the light-matter interaction in some situations and can be a nice solution to make sensors or nonlinear elements more efficient and more compact[20,21]. This is still an opened field for research and interesting demonstrations are expected in the coming years.

\section{References}

1. L. Thevenaz, "Slow and fast light in optical fibres," Nat Photon 2, 474-481 (2008).

2. K. Y. Song, M. Gonzalez Herraez, and L. Thevenaz, "Observation of pulse delaying and advancement in optical fibers using stimulated Brillouin scattering," Optics Express 13, 82-88 (2005).

3. Y. Okawachi, M. S. Bigelow, J. E. Sharping, Z. M. Zhu, A. Schweinsberg, D. J. Gauthier, R. W. Boyd, and A. L. Gaeta, "Tunable all-optical delays via Brillouin slow light in an optical fiber," Physical Review Letters 94, 153902 (2005).

4. K. Y. Song, M. G. Herraez, and L. Thevenaz, "Long optically controlled delays in optical fibers," Optics Letters 30, 1782-1784 (2005).

5. Z. M. Zhu, A. M. C. Dawes, D. J. Gauthier, L. Zhang, and A. E. Willner, "Broadband SBS slow light in an optical fiber," Journal of Lightwave Technology 25, 201-206 (2007).

6. R. Pant, M. D. Stenner, M. A. Neifeld, and D. J. Gauthier, "Optimal pump profile designs for broadband SBS slow-light systems," Optics Express 16, 2764-2777 (2008).

7. B. Zhang, L. Zhang, L. S. Yan, I. Fazal, J. Y. Yang, and A. E. Willner, "Continuously-tunable, bit-rate variable OTDM using broadband SBS slow-light delay line," Optics Express 15, 8317-8322 (2007).

8. C. Jauregui Misas, P. Petropoulos, and D. J. Richardson, "Slowing of pulses to c/10 with subwatt power levels and low latency using Brillouin amplification in a bismuth-oxide optical fiber," Journal of Lightwave Technology 25, 216-221 (2007).

9. M. Gonzalez-Herraez, K. Y. Song, and L. Thevenaz, "Optically controlled slow and fast light in optical fibers using stimulated Brillouin scattering," Applied Physics Letters 87, - (2005).

10. E. Shumakher, A. Willinger, R. Blit, D. Dahan, and G. Eisenstein, "Large tunable delay with low distortion of 10 Gbit/s data in a slow light system based on narrow band fiber parametric amplification," Optics Express 14, 8540-8545 (2006).

11. J. E. Sharping, Y. Okawachi, and A. L. Gaeta, "Wide bandwidth slow light using a Raman fiber amplifier," Optics Express 13, $6092-6098$ (2005).

12. A. Schweinsberg, N. N. Lepeshkin, M. S. Bigelow, R. W. Boyd, and S. Jarabo, "Observation of superluminal and slow light propagation in erbium-doped optical fiber," Europhysics Letters 73, 218-224 (2006).

13. J. T. Mok, C. M. de Sterke, and B. J. Eggleton, "Delay-tunable gap-soliton-based slow-light system," Optics Express 14, 11987-11996 (2006).

14. M. Gonzalez Herraez, K. Y. Song, and L. Thevenaz, "Arbitrary-bandwidth Brillouin slow light in optical fibers," Optics Express 14, 13951400 (2006).

15. K. Y. Song, and K. Hotate, "25 GHz bandwidth Brillouin slow light in optical fibers," Optics Letters 32, 217-219 (2007).

16. A. Zadok, A. Eyal, and M. Tur, "Extended delay of broadband signals in stimulated Brillouin scattering slow light using synthesized pump chirp," Optics Express 14, 8498-8505 (2006).

17. J. B. Khurgin, "Performance limits of delay lines based on optical amplifiers," Optics Letters 31, 948-950 (2006).

18. S. Chin, M. Gonzalez Herraez, and L. Thevenaz, "Zero-gain slow \& fast light propagation in an optical fiber," Optics Express 14, 10684$10692(2006)$.

19. T. Schneider, R. Henker, K. U. Lauterbach, and M. Junker, "Comparison of delay enhancement mechanisms for SBS-based slow light systems," Optics Express 15, 9606-9613 (2007).

20. M. Soljacic, and J. D. Joannopoulos, "Enhancement of nonlinear effects using photonic crystals," Nat Mater 3, 211-219 (2004).

21. Z. M. Shi, R. W. Boyd, D. J. Gauthier, and C. C. Dudley, "Enhancing the spectral sensitivity of interferometers using slow-light media," Optics Letters 32, 915-917 (2007). 